\title{
Blazar Optical Variability in the Palomar-QUEST Survey
}

\author{
Anne Bauer ${ }^{1,2}$, Charles Baltay ${ }^{1}$, Paolo Coppi ${ }^{1}$, Nancy Ellman ${ }^{1}$, Jonathan Jerke ${ }^{1}$, David \\ Rabinowitz $^{1}$, Richard Scalzo ${ }^{1}$ \\ anne.bauer@aya. yale.edu
}

\begin{abstract}
We study the ensemble optical variability of 276 FSRQs and 86 BL Lacs in the Palomar-QUEST Survey with the goal of searching for common fluctuation properties, examining the range of behavior across the sample, and characterizing the appearance of blazars in such a survey so that future work can more easily identify such objects. The survey, which covers 15,000 square degrees multiple times over 3.5 years, allows for the first ensemble blazar study of this scale. Variability amplitude distributions are shown for the FSRQ and BL Lac samples for numerous time lags, and also studied through structure function analyses. Individual blazars show a wide range of variability amplitudes, timescales, and duty cycles. Of the best sampled objects, $35 \%$ are seen to vary by more than 0.4 magnitudes; for these, the fraction of measurements contributing to the high amplitude variability ranges constantly from about $5 \%$ to $80 \%$. Blazar variability has some similarities to that of type I quasars but includes larger amplitude fluctuations on all timescales. FSRQ variability amplitudes are particularly similar to those of QSOs on timescales of several months, suggesting significant contributions from the accretion disk to the variable flux at these timescales. Optical variability amplitudes are correlated with the maximum apparent velocities of the radio jet for the subset of FSRQs with MOJAVE VLBA measurements, implying that the optically variable flux's strength is typically related to that of the radio emission. We also study CRATES radio-selected FSRQ candidates, which show similar variability characteristics to known FSRQs; this suggests a high purity for the CRATES sample.
\end{abstract}

Subject headings: BL Lacertae objects: general — galaxies: active — quasars: general

\footnotetext{
${ }^{1}$ Yale University, Department of Physics, P.O. Box 208120, New Haven, CT 06520-8120, USA

${ }^{2}$ Universitäts-Sternwarte München, Scheinerstr. 1, D-81679 München, Germany
} 


\section{Introduction}

Blazars are among the most variable objects known. Fluctuations occur on all observed timescales, and shifts of several magnitudes in optical brightness have been seen over the span of just months (see, e.g., Metcalf and Magliocchetti (2006)). The mechanisms behind these dramatic fluctuations are not well understood. A number of individual blazars have been carefully observed, revealing a large range of variability behavior. (e.g. Chatterjee et al. (2008); Raiteri et al. (2008)). Characteristic fluctuation timescales measured for specific blazars range from hours to years (e.g. Heidt and Wagner (1996); Ciprini et al. (2003); Kartaltepe and Balonek (2007)).

Traditionally, blazar variability has been studied through intensive monitoring of individual objects. With new wide-field surveys it is possible to take a complementary approach by studying a few measurements of many blazars. If the blazar class is completely homogeneous, the ensemble properties will be representative of each object. If the class is heterogeneous, then the ensemble properties will correspond exactly to none of the objects, but will nevertheless be informative in statistically describing the sample. The ensemble variability of type I quasars has been studied in this manner (e.g. Vanden Berk et al. (2004); Bauer et al. (2009)) and compared with more detailed work on individual objects (e.g. Collier and Peterson (2001)) in order to understand better the causes of quasar variability and the range of physical processes at work in these systems. Observations of individual AGN suggest that blazar variability exhibits much more diversity of amplitude and timescale than quasar variability. No large ensemble study of blazar variability has yet been published to study this effect, however, due to the lack of repeated measurements over large enough areas of sky. Wide-field surveys such as Palomar-QUEST are able to observe large numbers of blazars and begin to study the collective variability properties of the class, and to examine the extent of heterogeneity within the sample.

The characterization of blazar variability as seen by several measurements over a wide-field survey is useful for another purpose: as a tool for using variability to identify blazars in such surveys. Knowledge of blazar physics is limited in part because relatively few blazars are known; therefore the discovery of more blazars is important for a better understanding of the objects' behavior. Furthermore, nearly all blazars currently studied have been selected by their flux ratios in the radio and/or X-ray bands, possibly contributing to significant selection effects in the ensemble. As well as typically exhibiting strong radio and X-ray emission, blazars are also some of the most optically variable objects in the sky. By studying the appearance of blazars as a class in sparsely sampled variability surveys we can perhaps facilitate the discovery of many more in current surveys like Palomar-QUEST, as well as imminent surveys such as Pan-STARRS.

Blazars are currently understood to be active galactic nuclei (AGN) with jets, where the jet axis is aligned along the observer's line of sight. Their flux is therefore dominated by beamed jet emission across the electromagnetic spectrum. The broadband spectral energy distributions (SEDs) of blazars exhibit two broad peaks, one at lower energies (ranging in frequency from radio to $\mathrm{X}$-rays) and one at higher energies (from X-rays to $\mathrm{TeV}$ gamma rays). The low-energy peak 
is commonly attributed to synchrotron emission from high-energy particles accelerated in regions of shocked gas in the jet. These high-energy particles can also reprocess synchrotron radiation from the low-energy peak, thermal photons from the accretion disk, or other external photons via Compton upscattering to form the high-energy peak. Hadron synchrotron radiation or decay may also contribute to the high-energy peak (see, e.g., Mücke and Protheroe (2001)).

Blazars are often divided into subcategories of BL Lac objects and flat spectrum radio quasars (FSRQs), based in part on the absence or presence, respectively, of broad optical emission lines superimposed on the characteristic jet continuum emission. These broad lines are accretion disk emission reprocessed by energetic gas that is close to the system's central black hole. These emission lines are visible in FSRQs but are most prominent in type I quasars, which are AGN aligned somewhat off-axis such that the observer views flux predominantly from the accretion disk. Reviews of the typical constituent parts of an AGN and the orientation-based theory of AGN unification linking quasars and blazars can be found in, for example, Urry and Padovani (1995) and Peterson (1997).

FSRQs and BL Lacs are believed to differ not just in their emission lines, but also in the structure and power of their jets. Radio galaxies, or AGN with misaligned jets, are divided into two basic categories: Fanaroff-Riley types I and II (FR I and FR II) (Fanaroff and Riley (1974)). FR I jets generally have lower energies and are spatially limited, with the power concentrated close to the center of the galaxy. FR II jets are more energetic and extend much further out, with the most luminous areas in lobes far from the center of the galaxy. FSRQs and BL Lacs are believed to belong to the same population as FR IIs and FR Is, respectively. FSRQs are more luminous than BL Lacs, as FR IIs are compared to FR Is. Furthermore, the relative number counts of FSRQs with respect to FR IIs, and BL Lacs with respect to FR Is, are consistent with the blazars being those radio galaxies that are by chance aligned to within $\sim 15^{\circ}$ of the line of sight (Urry and Padovani (1995)). This blazar/radio galaxy unification model implies that FSRQs have higher energy jets than BL Lacs.

Because of the morphological, energetic, and spectral differences between FSRQs and BL Lacs, we study the two types separately. Distinctions between the optical variability of the groups can improve our understanding of the fundamental differences between these subclasses.

The dramatic variability of blazar flux has several possible causes. For example, the intermittent nature of shocks in the jet produces highly variable emission. Much work has been done to model shock dissipation mechanisms in order to understand blazars' variability and energy distributions. However, current models are not well constrained; even a single model framework can yield many possible variability characteristics (see, e.g., Li and Kusunose (2000)). It is also uncertain how the sources of different wavelength emission are physically related to each other, for example if the radio, optical, and/or X-ray flux are generated in the same jet location. Multiwavelength monitoring of several blazars has revealed some cases in which radio, optical, and X-ray flares appear to have a common source; other cases show one band's emission to be unaffected by another's 
fluctuations (see, e.g., D’Arcangelo et al. (2007); Chatterjee et al. (2008); Marscher et al. (2008); Bonning et al. (2009)).

Variability may also be due to geometric effects. Shocks and instabilities can kink the jet, changing the beaming direction with respect to our line of sight and therefore affecting how much jet flux reaches us. Or, beaming direction changes can be due to the precession of the black hole if it is in a binary system. Precession is not thought to be a dominant source of variability, as most blazars studied have clearly aperiodic flux patterns. Some blazars, however, do show somewhat regular variability. Ciaramella et al. (2004) found periodic features in the radio emission of 5 out of 77 blazars studied, with periods ranging from about 3 to 9 years.

The Palomar-QUEST Survey scans 15,000 square degrees of sky multiple times, thereby repeatedly observing a large number of blazars in optical wavelengths. Those with consistent classification, precise redshift measurements, and multiple good-quality Palomar-QUEST measurements number 362 in total: 86 BL Lacs and 276 FSRQs. Each blazar has, on average, roughly four observations. By compiling and comparing sparse variability measurements of an unprecedented number of blazars we can study the typical variability behavior of the blazar class and the range of common fluctuations.

In $\oint 2$ we briefly describe the Palomar-QUEST Survey. 3 introduces the samples of BL Lacs and FSRQs used in this analysis. $\$ 4$ discusses the techniques which we use to analyze timescales of variability. Results are presented in 95 and discussed in 96 , and we conclude in $\$ 7$.

\section{The Palomar-QUEST Survey}

The Palomar-QUEST Survey is a large area optical survey which uses the 48" Samuel Oschin Schmidt Telescope at Palomar Observatory. Over the span of 3.5 years we have observed 15,000 square degrees of sky multiple times with seven optical filters: Johnson UBRI and SDSS r'i'z'. The sky area has been covered typically 4-5 times in each filter, with the time between passes ranging from hours to years. The data were taken using the QUEST2 Large Area Camera, which was built for the survey. The camera is made up of $112 \mathrm{CCDs}$, covering in total $4.6^{\circ} \times 3.6^{\circ}$, which are arranged into 4 rows such that a separate filter can be placed over each row. The camera was used in driftscan mode to take the data. In this mode, an 8 hour night of observing yields $\sim 500$ square degrees of data in 4 filters with an exposure time of roughly 140 seconds. The camera is described in detail in Baltay et al. (2007).

The Palomar-QUEST data are processed using custom-written software. Objects are detected by fitting flux peaks with an empirical point spread function (PSF) model, which allows for accurate treatment of close neighbors. Photometry is performed using PSF and also aperture fitting, with the PSF measurements used as the primary results. Astrometry is calculated with respect to the USNO A-2.0 catalog (Monet et al. (1998)), and is accurate and precise to 0.1". This processing software is described in detail in Andrews et al. (2008). 
The calibration of the Palomar-QUEST data for variability work involves five basic steps. First, zeropoints are applied to correct for sensitivity variations across each individual chip as well as for nonlinearities in each chip, and also for different response levels from chip to chip. These zeropoints were calculated once, and correct for the constant response characteristics of the CCDs. Next, for each 1/16 square degree of sky under consideration, a photometrically stable Palomar-QUEST scan is chosen to be used as the calibration standard. An RA-dependent correction is applied to all scans overlapping the standard to account for changing weather conditions. A declinationdependent calibration corrects for spatially-dependent factors such as scattered light in the camera. Finally, strict quality cuts are made to eliminate poor measurements and substandard data regions. Because it is important for variability work to have as many comparable measurements of an object as possible, we calibrate together data from the Johnson R and SDSS r' filters to form an "Rr" bandpass. Since the R and r' wavelength ranges are similar, the color terms introduced by this combination are small. The calibration yields a systematic error of $0.7 \%$ for typical pointlike objects in the "Rr" band, which are the data used in this work. For a thorough explanation of the variability calibration see Bauer et al. (2009), referred to henceforth as Paper I.

\section{The Blazar Sample}

We have studied a set of blazars accumulated from various sources: Stickel et al. (1991), Hewitt et al. (1993), Collinge et al. (2005), Donato et al (2005), Sowards-Emmerd et al. (2005), Veron-Cetty and Veron (2006), Massaro et al. (2007), Turriziani et al. (2007), and Healey et al. (2008). These lists have many objects in common. In total, in the Palomar-QUEST Survey area, including only objects whose positions are known to roughly 1 arcsecond, whose redshifts have been measured, and which have multiple well-calibrated Palomar-QUEST observations, we have collected 94 unique BL Lacs and 278 unique FSRQs. There are also four objects that were classified by some studies as BL Lacs and by others as FSRQs. This highlights the fact that a blazar's broad emission lines often vary in prominence over time, sometimes to the point of making the classification unreliable. We did not include the four ambiguous objects in our study. There may be more such blazars that remain in the sample, thereby confusing the results. Since only about $1 \%$ of the sample is doubly classified, however, we expect the effect to be small.

A histogram of the number of good quality QUEST Rr observations of each blazar is shown in figure 1, The redshift distribution of the blazar sample is shown in figure 2, Note the difference between the BL Lac and FSRQ redshift distributions, due to the fact that BL Lacs are intrinsically fainter than FSRQs, and therefore can be detected by QUEST only at lower redshifts (see, e.g., Padovani et al. (2007)). The blazar distribution is suppressed at redshifts lower than 0.2 . The catalogs from which we took the objects do not have this limit; there are many blazars, particularly BL Lacs, with lower redshifts. However, the variability calibration of the Palomar-QUEST data rejects measurements of objects that appear spatially extended, as our flux measurements of extended objects are not reliable. The details of and motivation for this cut are discussed in Paper 
I. Most of the blazars with redshifts below 0.2 appear extended in our data and therefore do not yield good quality measurements. We therefore remove all blazars from our list that have redshifts less than 0.2. This cut eliminates 8 BL Lacs and 2 FSRQs, leaving us with a final sample of $86 \mathrm{BL}$ Lacs and 276 FSRQs.

\section{Analysis Techniques}

\section{1. $\quad \mathbf{S F}(\tau)$}

The structure function is a statistical quantity often used to study the variability of AGN (see, e.g. Paper I, Vanden Berk et al. (2004)). It can be defined as

$$
S F(\tau)=\sqrt{<[m(t)-m(t-\tau)]^{2}>-<\sigma^{2}>}
$$

where $m(t)-m(t-\tau)$ is the difference in measured magnitudes of an object at two epochs separated by rest frame time $\tau$. $\sigma$ is the measurement error on the magnitude difference term. $<X>$ denotes the mean value of $X$ over the set of objects. We perform three iterations of $3 \sigma$ clipping prior to averaging the data. The structure function is a measure of the intrinsic variability of an object, and as it is a function of rest frame time lag $\tau$ it is useful in determining characteristic timescales of variability.

The shape of the structure function vs. $\tau$ depends on the sample's variability in certain characteristic ways. For short timescales, if the intrinsic variability is less than the measurement

noise, then the structure function will plateau. For timescales longer than the maximum timescale characteristic of the variability, the plot will also plateau. Between these possible plateaus the structure function rises with a shape determined by the details of the system. For example, if the variability is characterized by a power law frequency distribution then the structure function will also yield a power law shape, with an index directly related to the frequency dependence. Other variability patterns can produce more complicated structure functions. In general, changes in the shape of the structure function indicate timescales important to the variability mechanism.

\section{2. $\mathrm{V}(\tau)$}

Because it averages over variability amplitudes, the structure function does not describe the range of variability behavior observed in a sample. If two samples have a similar structure function it is not clear if the distributions of variability amplitudes are truly similar, or if they only have similar mean values. To show the range of blazar behavior explicitly, we calculate, for each pair of measurements for each object,

$$
V(\tau)=\sqrt{[m(t)-m(t-\tau)]^{2}-\sigma^{2}}
$$




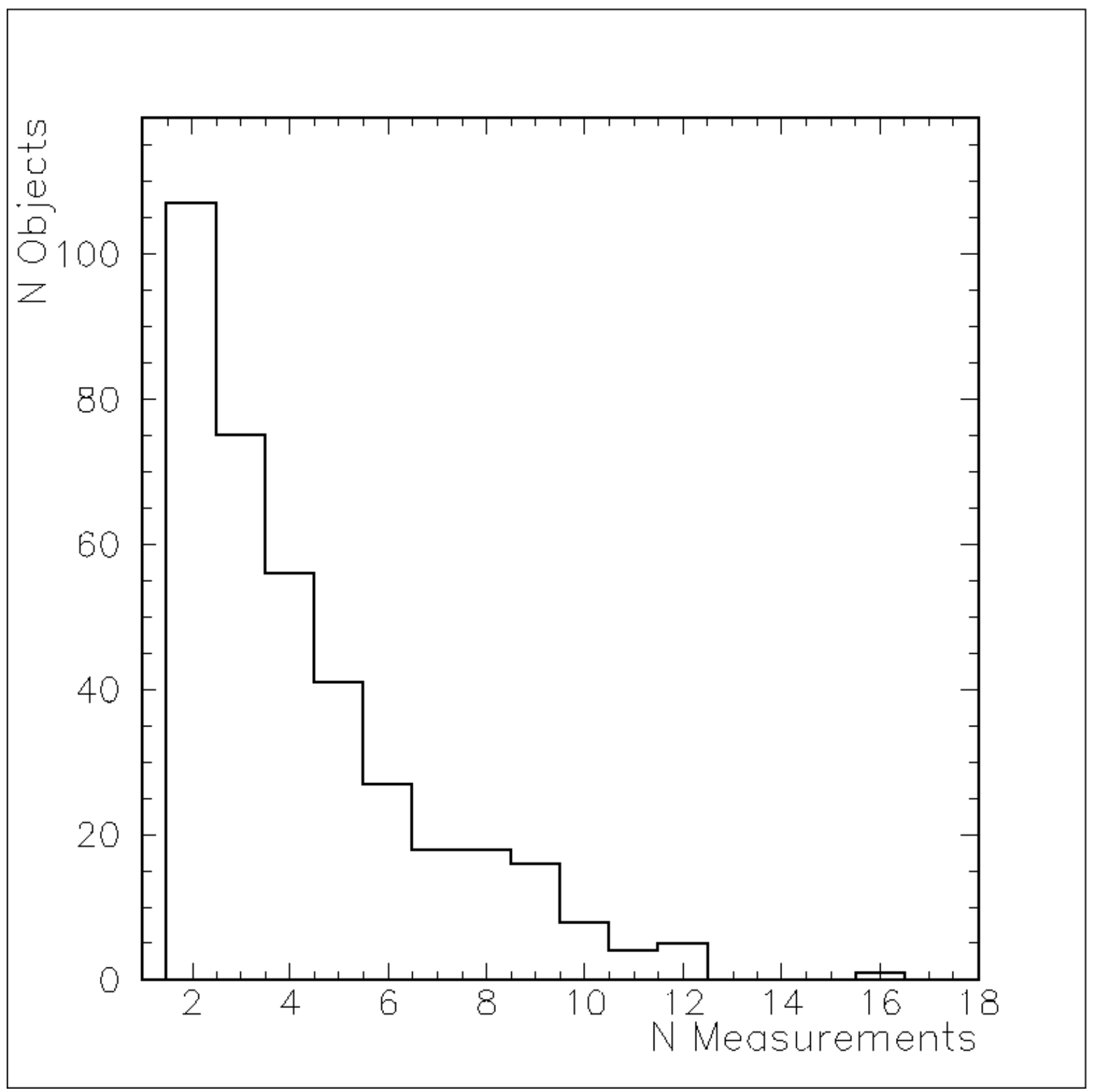

Fig. 1. - Number of blazars with $\mathrm{N}$ good Rr measurements. 


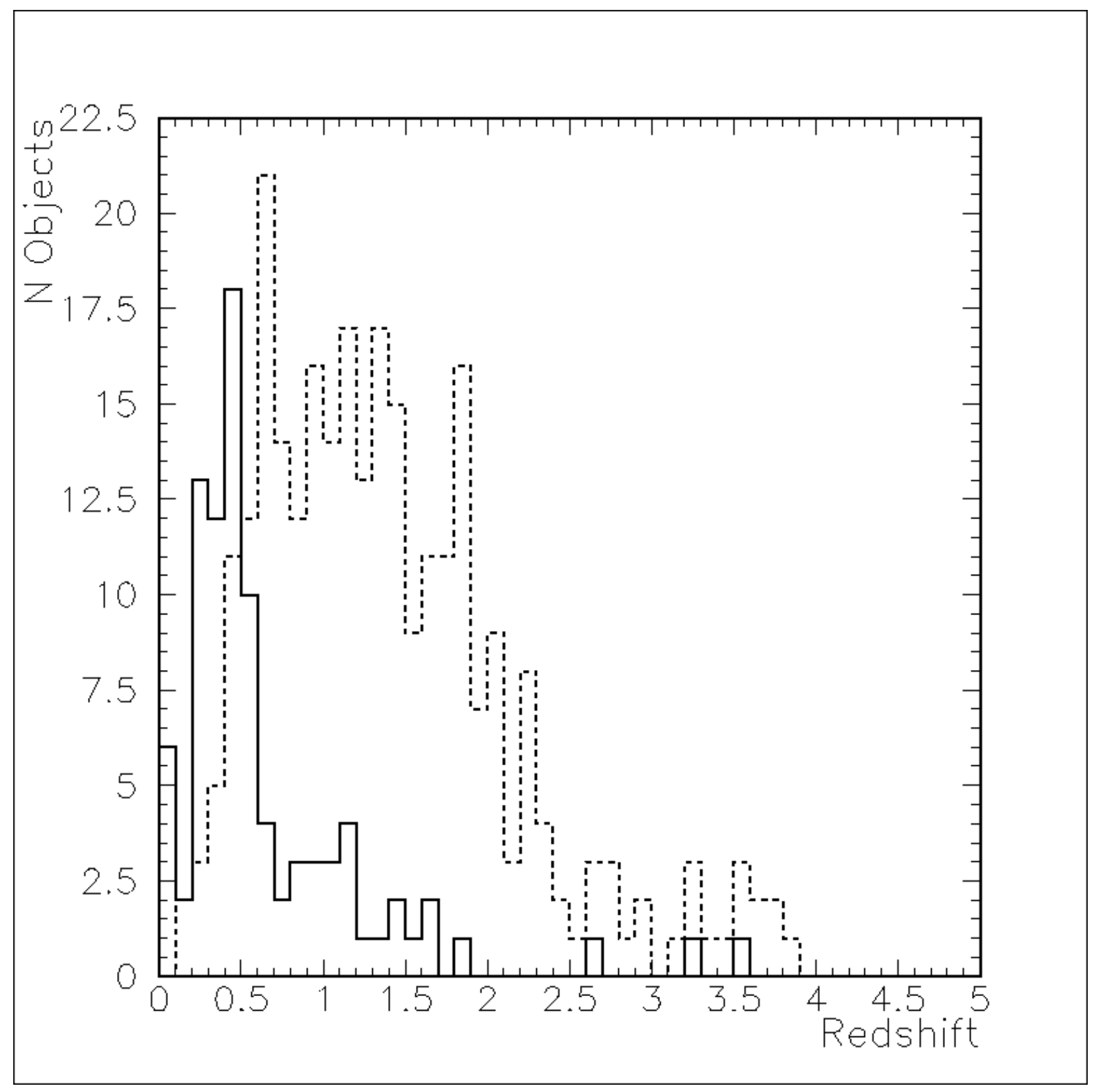

Fig. 2.- Redshift distribution of the blazars. Solid line: BL Lacs. Dashed line: FSRQs. 
where $m(t)$ and $m(t-\tau)$ are two magnitude measurements of the same object separated by rest frame time lag $\tau$, and $\sigma$ is the uncertainty on the magnitude term. This quantity is similar to the structure function except it is not an average over the sample; a blazar with $N$ magnitude points will yield $N \times(N-1) / 2$ separate values of $V$. For measurement pairs where no variability is seen and $[m(t)-m(t-\tau)]^{2}<\sigma^{2}, V$ is set to zero.

\section{Results}

\subsection{Distributions of $\mathrm{V}(\tau)$}

Figures 3 and 4 show histograms of $V$ for FSRQs and BL Lacs, respectively, each split into nine $\tau$ bins. Also shown in each plot as a dashed line are the corresponding data for the type I quasar sample studied in Paper I. The blazar and quasar histograms are each normalized to a total of 100 objects so that the two are easily comparable by eye.

These plots show qualitative differences between the samples. Both BL Lacs and FSRQs show excess high amplitude variability compared to the quasars on all timescales. Both BL Lacs and FSRQs often show a low amplitude variability peak similar to that of quasars, particularly at shorter timescales. This quasar-like peak is more pronounced in FSRQs than in BL Lacs. The FSRQs show more variability at the highest amplitudes, with $V \gtrsim 1$ magnitude, than the BL Lacs.

\subsubsection{Effects of Sampling on $V(\tau)$}

Repeated QUEST measurements of each blazar in the sample are not made at regular intervals. For example, sometimes measurements were made of the same sky area three months apart. However, it is impossible to observe the same sky area from the ground at a six month interval. Because each of the nine $\tau$ bins over which we measure $V$ are not populated by measurements with a uniform $\tau$ distribution, the results in figures 3 and 4 may be skewed by the details of our sampling cadence. To study this effect, we have examined how the data's windowing function alters $V$ histograms measured from one blazar with unusually good historical sampling: 3C 273. Ideally, we would like to study the effects of sampling on a "typical" blazar lightcurve. However, sufficient data have not been collected for enough individual blazars over the timescale of the QUEST survey to empirically determine "typical" behavior. We do not wish to interpolate between sparse measurements of a large number of blazars to calculate an averaged lightcurve, as an interpolation between distant points would not reproduce the short term variability we know to exist in blazars. Furthermore, theoretical models of synchrotron emission lack the short-term variability commonly

observed in blazar lightcurves (see, e.g., Lindfors et al. (2006)), and are therefore not sufficient as models for studying how sampling affects the measurement of such variability. We therefore take 3C 273, a blazar with exceptional coverage over several decades, as an example object to probe the 


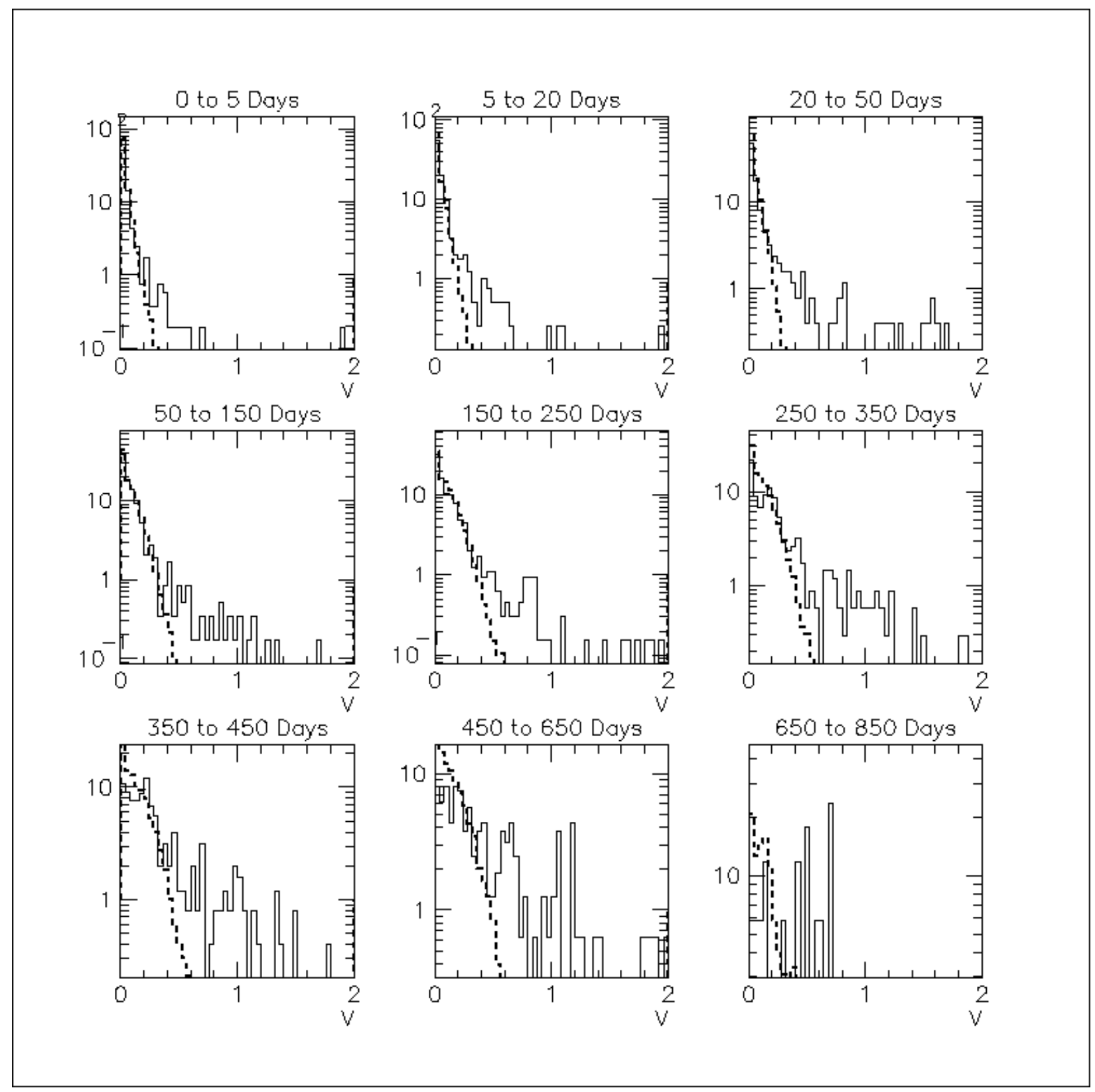

Fig. 3.- Variability $V$ histograms of FSRQs (solid line) and quasars (dashed line) for 9 different time lag bins. 


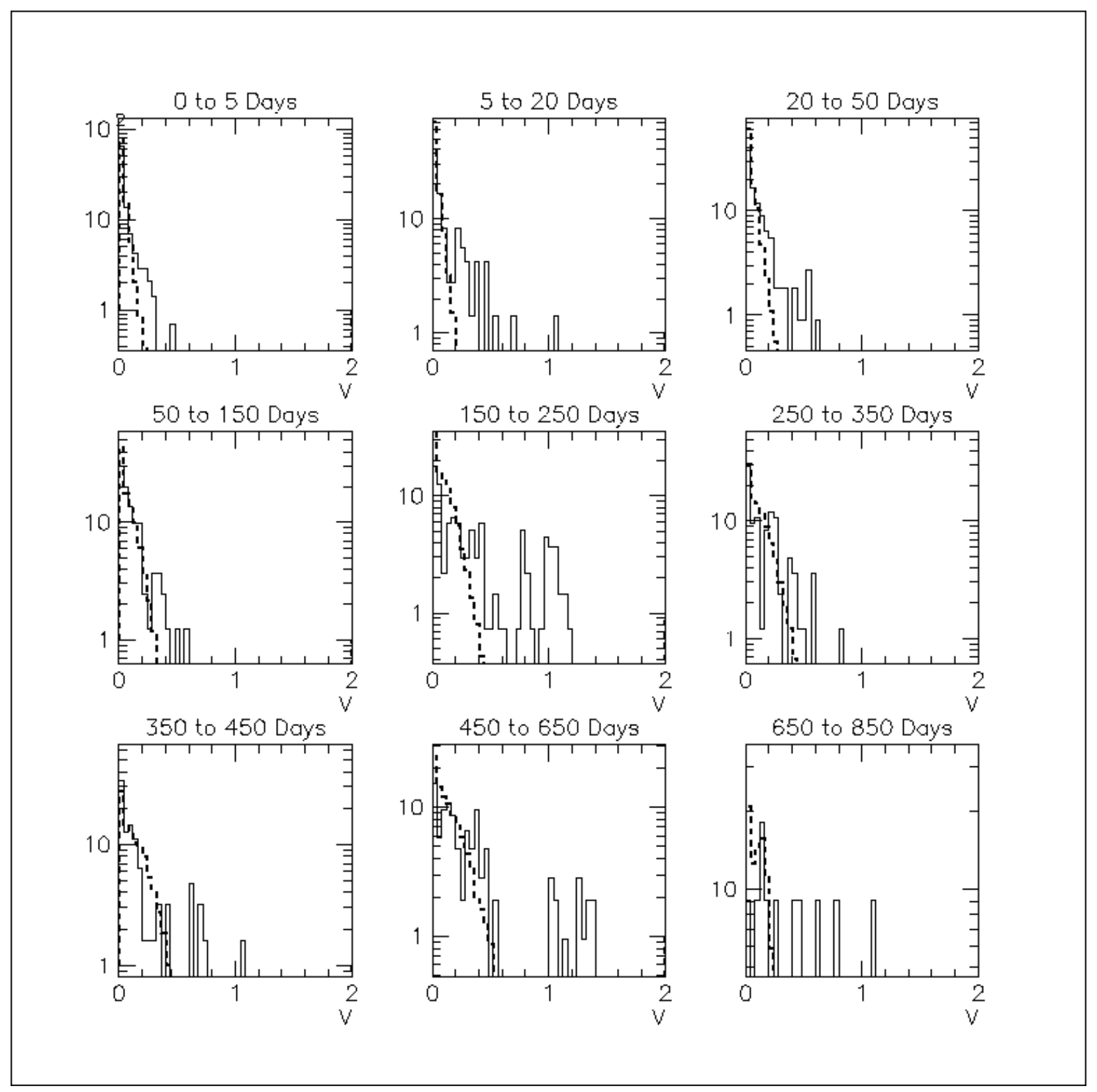

Fig. 4.- Variability $V$ histograms of BL Lacs (solid line) and quasars (dashed line) for 9 different time lag bins. 
effects of windowing in the QUEST data. Because the data cadence's effects on a measurement depend on the details of objects' lightcurves, one blazar is not sufficient to completely describe the effects of windowing on our sample. However, if the results are strongly and systematically skewed by our uneven data cadence, we expect every blazar lightcurve to be influenced by the effect. 3C 273 should therefore be a useful diagnostic to detect significant windowing effects in the results. For this work we use V-band data available at http://isdcul3.unige.ch/3c273.

To construct $V$ histograms from 3C 273 data that are not affected by windowing, we use a subset of the available measurements which are distributed nearly evenly in $\tau$. First, $V$ is calculated for each pair of measurements in the lightcurve. This yields a large number of $V$ s with a non-uniform distribution of $\tau$ ranging from hours to nearly 40 years. We then bin the $\tau$ values in units of one day (bins of less than 1 day would not all be populated since the measurements do not have spacings of $\sim 0.5$ day). From each bin we randomly choose a $V$ measurement with which to populate the evenly-sampled $\mathrm{V}$ histograms. The data are split into nine $\tau$ histograms as in figures 3 and 4 in order to mimic the QUEST results. For all but the first $\tau$ histogram, the one-day binning in $\tau$ introduces a small $\tau$ error compared to the length of the measurements' time lags. For the 0-5 day $\tau$ histogram, this procedure will not probe the effects of the data cadence as finely, as each bin width comprises $20 \%$ of the $\tau$ range. $V$ histograms constructed using the nearly evenly-distributed 3C 273 data are shown as the thick dashed lines in figures 5 and 6.

To assess the effects of the QUEST data cadence, we recalculate the $V$ histograms for a subset of the 3C 273 data which adheres to the QUEST sampling rate. The $\tau$ distribution for the QUEST blazar measurements is calculated and binned into one-day bins. For each one-day bin, for each QUEST measurement pair we select at random a $V$ from the same $\tau$ bin of 3C 273 data. $V$ histograms constructed using this QUEST-sampled 3C 273 dataset are shown as the solid lines in figures 5 and 6, the FSRQ sampling is used for figure 5, and the BL Lac for figure 6, In both figures, both datasets are normalized to a total of 100 objects in order to be easily comparable by eye.

Figures [5] and 6] show that the evenly-distributed and QUEST-distributed 3C 273 data yield similar $V$ histograms. The exact histogram shapes depend on the random $V$ values chosen from each 3C 273 bin. To quantitatively compare the distributions we create 100 such evenly-sampled and QUEST-sampled sets and compare them, separately for each $\tau$ interval, using a KolmogorovSmirnov (K-S) test. For this comparison we ignore measurement pairs with $[m(t)-m(t-\tau)]^{2}<\sigma^{2}$ rather than setting $V=0$ in those cases, as the K-S test is sensitive to the shape of the distribution and will be strongly affected by an artificially sharp peak at $V=0$. The $100 \mathrm{~K}-\mathrm{S}$ results for each $\tau$ histogram are averaged to find a best estimate of the probability that the QUEST-sampled histograms come from the same underlying distribution as the evenly-sampled histograms. The results of the K-S tests are shown in table 1, It is not possible to distinguish the two datasets as coming from different distributions. We therefore conclude that the details of the QUEST sampling cadence do not significantly affect the shapes of the $V$ histogram results for our sample blazar. 


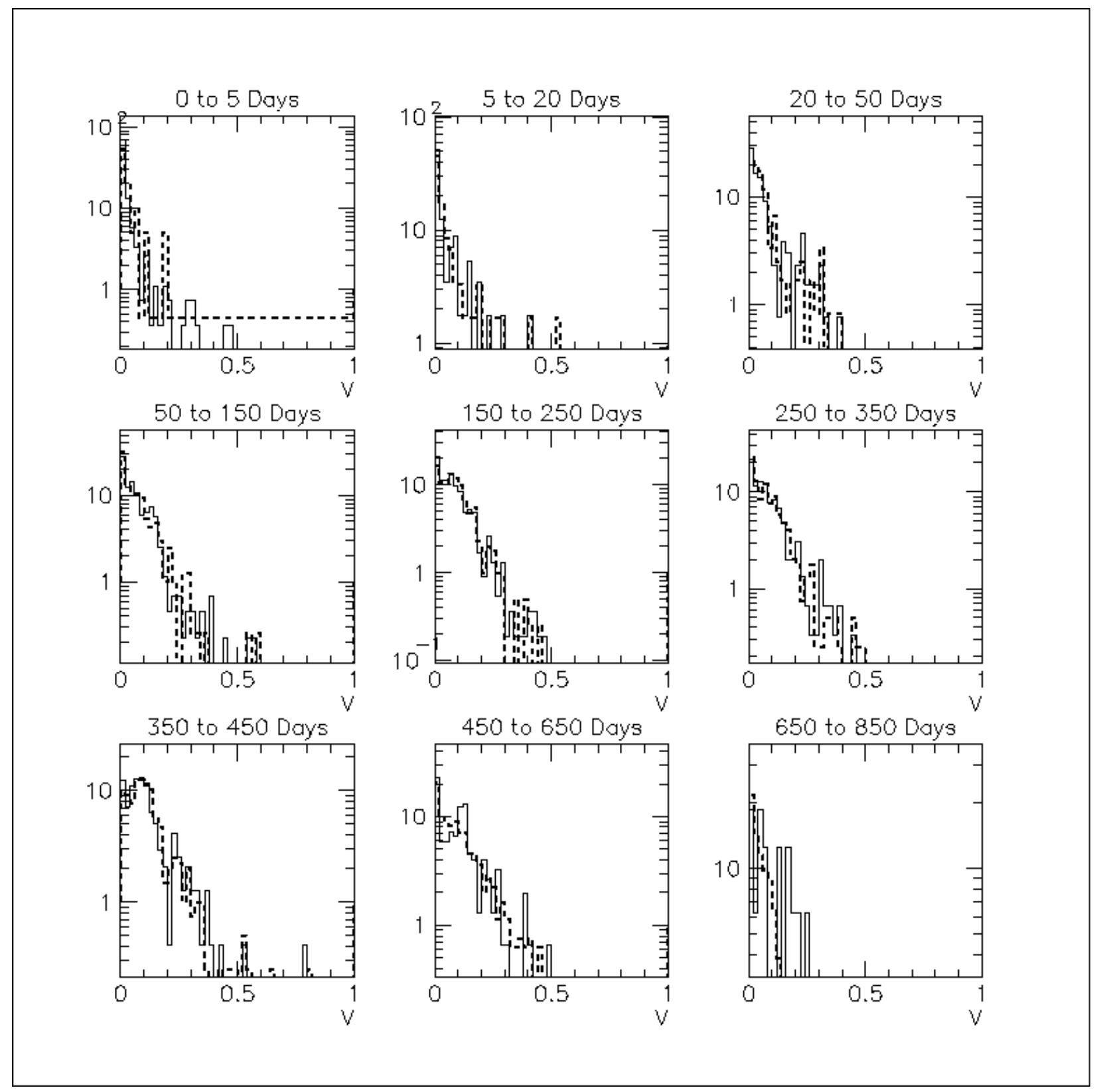

Fig. 5. $-V$ histograms for 3C 273 using data evenly distributed in $\tau$ (dashed line) and data distributed with the QUEST FSRQ sampling cadence (solid line). 


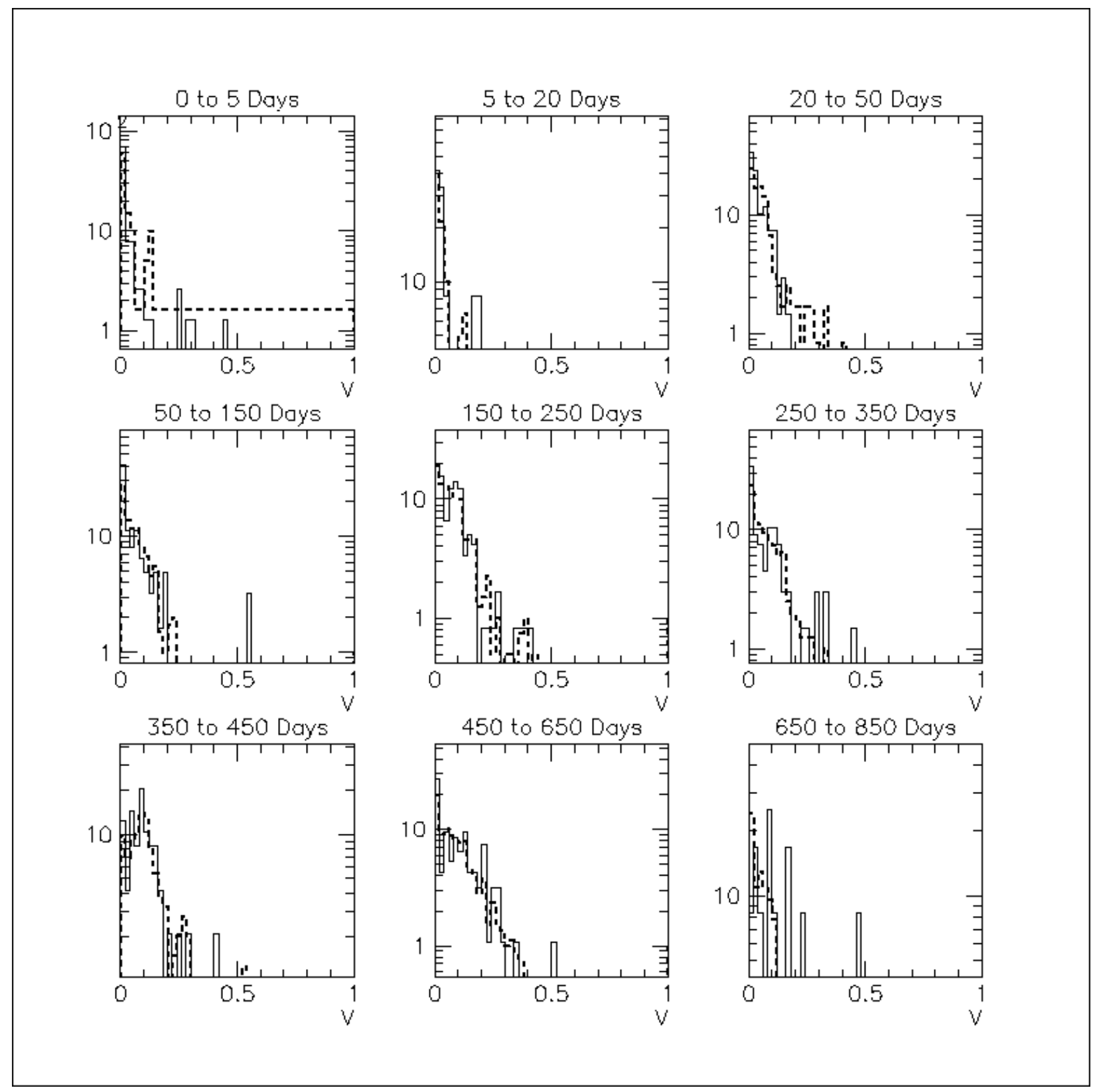

Fig. 6. $-V$ histograms for 3C 273 using data evenly distributed in $\tau$ (dashed line) and data distributed with the QUEST BL Lac sampling cadence (solid line). 


\section{2. $\quad \mathbf{S F}(\tau)$ vs. $\tau$}

The structure functions for the FSRQ and BL Lac samples, plotted versus rest frame time lag between measurements, are shown as the solid points in panels (a) and (b) of figure 7 . The errors on the structure function points are the $1 \sigma$ standard deviation of values obtained by analyzing subsets of the total sample. The structure function shapes are qualitatively different from that of the type I quasar structure function, measured in Paper I using Palomar-QUEST and shown for reference as the $\times \mathrm{s}$ in figure 7 . The turnover of the quasar structure function at the longest time lags is a known windowing effect, discussed in detail in appendix A of Paper I. The steady rise of the quasar structure function up to the windowing turnover indicates a lack of characteristic variability timescale in the quasar data. This is not the case for either the FSRQs or the BL Lacs, which show distinct peaks and changes in shape. The FSRQ and quasar structure functions are consistent, however, between timescales of roughly 70 and 250 days. The structure functions further display the results seen in the $V$ histograms, that both BL Lacs and FSRQs are at least as variable as type I quasars on all timescales measured by the survey.

Because the structure function presents the mean variability of the ensemble, it is sensitive to outlying data points. The data undergo three iterations of $3 \sigma$ clipping prior to the averaging, but there is no requirement that the result converge. Because blazars have significant high-variability tails, the structure function is not the ideal quantity to describe the variability, as it can easily be skewed by statistical variations of a poorly sampled, high $\Delta m$ tail. Nevertheless, as the structure function has been used to study type I quasars as well as individual blazars (e.g. Heidt and Wagner (1996); Kartaltepe and Balonek (2007)), we present the results here to allow for comparisons with other work.

\section{3. $\quad \mathbf{S F}(\tau)$ Modelling}

To demonstrate that the difference seen between the structure functions of quasars and blazars is not due to the data statistics and cadence, we generate lightcurves with power spectral distribution (PSD) proportional to $f^{-1.71}$, which fit the quasar results well in Paper I. We then analyze the model lightcurves using only simulated data points from epochs for which we have real data. The resulting simulated structure functions, thereby subjected to our blazar window functions, are shown in figure 8, As expected, the simulated structure functions rise roughly as a power law, with plateaus at low and high time lags due to measurement noise and edge effects. The low statistics cause the BL Lac simulated result to be noisy, yet it is consistent with the expected shape.

These simulated structure functions are qualitatively unlike the data; a power law frequency dependence does not yield the peaks and changes in slope observed in the blazar results. In fact, assigning one model lightcurve to every blazar consistently produces a structure function with

gradual rises and without discrete peaks. For example, a lightcurve model with $\sim 40$ day flares yields a structure function that rises until $\tau \sim 40$ and remains constant thereafter. If additional, longer 


\begin{tabular}{|l|l|l|}
\hline$\tau$ (Days) & FSRQ P & BL Lac P \\
\hline $0-5$ & 0.33 & 0.48 \\
$5-20$ & 0.43 & 0.38 \\
$20-50$ & 0.48 & 0.53 \\
$50-150$ & 0.49 & 0.58 \\
$150-250$ & 0.59 & 0.52 \\
$250-350$ & 0.46 & 0.49 \\
$350-450$ & 0.59 & 0.56 \\
$450-650$ & 0.27 & 0.39 \\
$650-850$ & 0.52 & 0.48 \\
\hline
\end{tabular}

Table 1: K-S probabilities P that the $V$ histograms from evenly-distributed and QUEST-distributed 3C 273 data come from the same underlying distribution. Calculated separately for FSRQ and BL Lac sampling cadences.
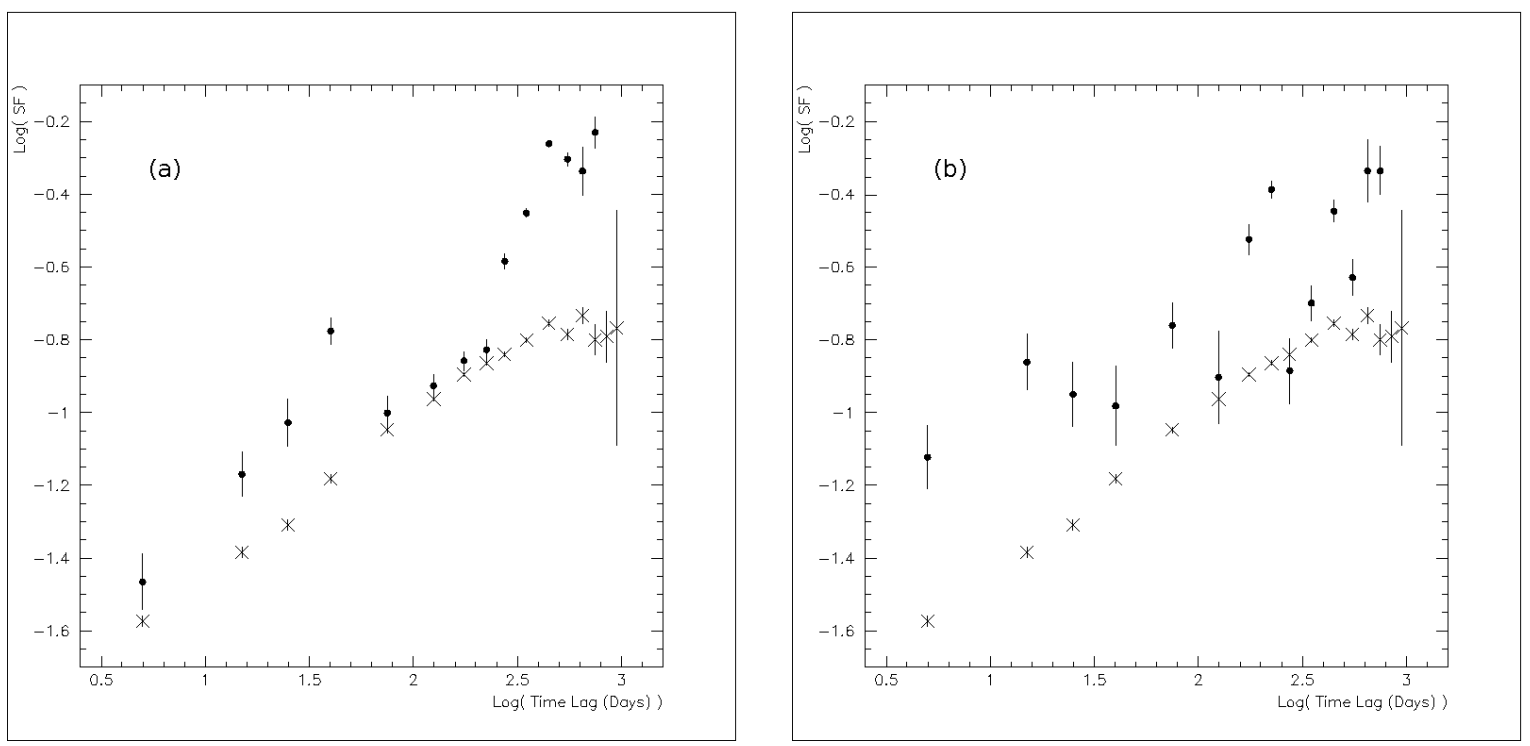

Fig. 7.- Structure function vs. rest frame time lag for FSRQs (a) and BL Lacs (b). Plotted on a log-log scale. Filled circles are blazar data, $\times$ symbols are quasar data from Paper I. 
term flares are added, the structure function continues rising, with a change of slope around 40 days but no discrete peak. The peaky, choppy nature of the FSRQ and BL Lac structure functions indicates that the variability characteristics differ significantly across the sample. This can be due to individual objects behaving uniquely, therefore making up an ensemble with a range of typical behaviors. Or, the mean variability can be disproportionately affected by a few measurements with high variability amplitude. Simulations using lightcurve features such as fast shifts in amplitude for only some objects do indeed create noisy, peaked structure functions; however the simulation results vary greatly across instantiations. Because each blazar is observed typically only four times, it is not possible to measure specific lightcurve features directly from the data. Furthermore, the complicated structure functions do not allow us to identify blazar lightcurve features such as the flares' exact durations or recurrence rates. Instead, the modelling shows us that the blazar lightcurves show average variability that is indeed larger than that of type I quasars on both short and long timescales, and that the blazars' fluctuations over the timescales measured cover a wide range of amplitudes.

We can further investigate the influence of the window function through the structure function of $3 \mathrm{C} 273$. We use the same method as in section 5.1.1 to generate evenly-sampled and QUESTsampled datasets for $3 \mathrm{C} 273$. The structure functions calculated from these datasets are shown in figure 9, using the FSRQ sampling cadence in panel (a) and the BL Lac cadence in panel (b). The hollow circles indicate the evenly-sampled data; the asterisks indicate the QUEST-sampled data. Error bars are determined using subsets of the total sample, as in the main analysis. The structure functions are consistent with each other, showing that windowing does not significantly alter the structure function results for the blazar.

Incidentally, 3C 273 is an example of an individual blazar that does not adhere to the description of its class's ensemble variability. This object typically does show strong short timescale variability, but over the timescales studied its average variability amplitude does not increase dramatically with $\tau$.

\subsection{Effects of Radio Jet Velocities}

MOJAVE (Lister and Homan (2005)) has monitored hundreds of blazars' radio brightnesses and polarizations using the VLBA. Their sample includes 5 of our BL Lacs and 31 of our FSRQs. The maximum apparent FSRQ jet speeds $\beta_{\text {app }}$ measured by MOJAVE 1 are shown in figure 10 in units of the speed of light. Table 2 lists the median $V$ values for FSRQs with different ranges of $\beta_{a p p}$. The range of $V$ increases with $\beta_{a p p}$, with FSRQs with higher $\beta_{a p p}$ showing more high amplitude variability.

\footnotetext{
${ }^{1}$ Data available at http://www.physics.purdue.edu/astro/MOJAVE/
} 

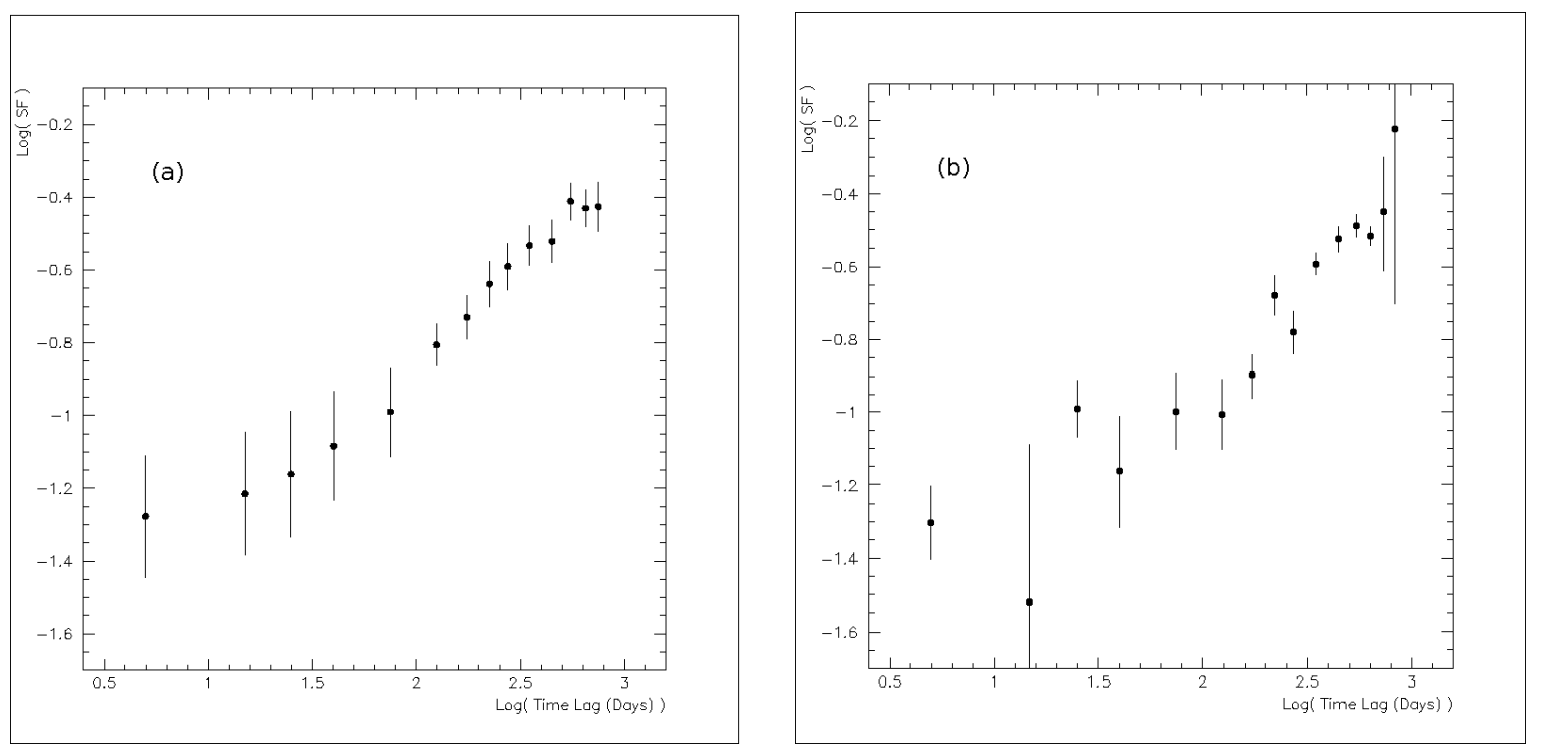

Fig. 8. - Simulated FSRQ (a) and BL Lac (b) structure functions using $P S D \propto f^{-1.71}$ frequency dependence
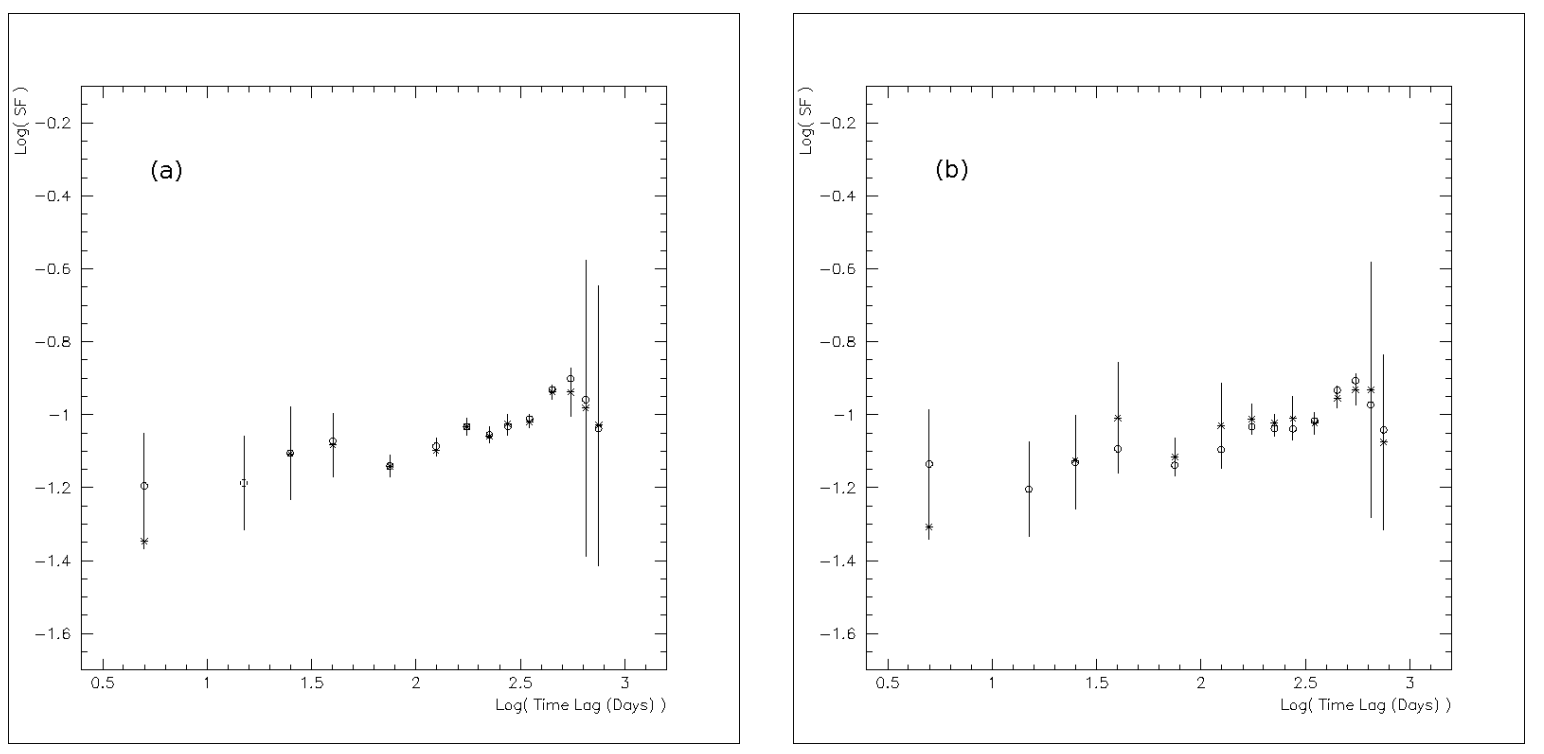

Fig. 9.- Structure functions for 3C 273 using evenly sampled (hollow circles) and QUEST sampled (*) data. (a): FSRQ sampling; (b): BL Lac sampling. 


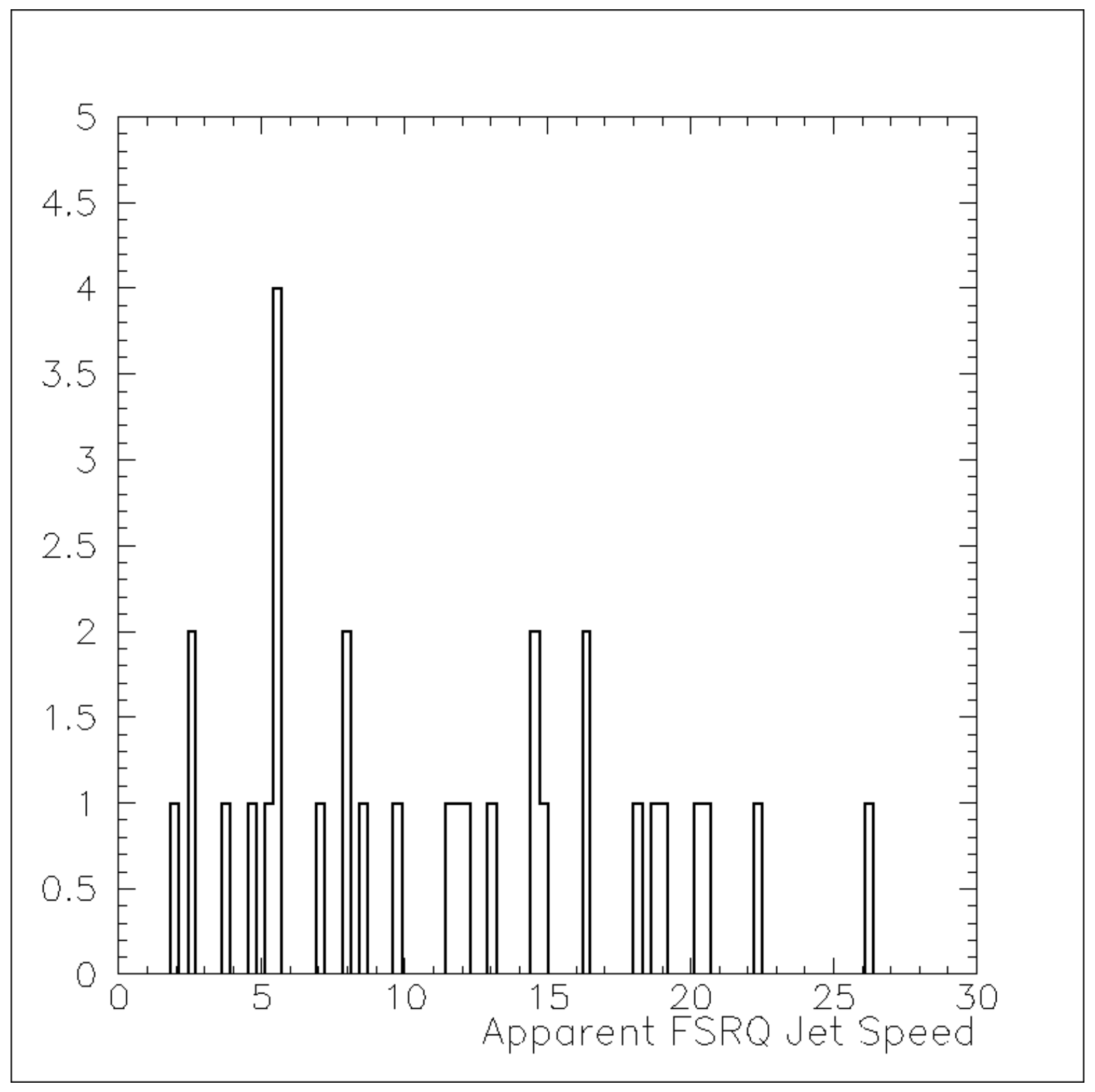

Fig. 10.- $\beta_{a p p}$ for 31 FSRQs, measured by MOJAVE. 


\subsection{Variability of CRATES Objects}

CRATES (Healey et al (2007)) presents a very large sample $(\sim 11,000)$ of FSRQ candidates selected in the $8.4 \mathrm{GHz}$ radio band. We compare the optical variability of these candidates with that of the confirmed FSRQ sample. The samples differ in two important ways: the CRATES candidates have unknown redshifts and include fainter objects than the FSRQs. To compare variability timescales, we use the observer frame time lag $t$ rather than $\tau$ and recalculate the variability distributions for the known FSRQs also using the observer's frame time lag between measurements. If the distributions of object redshifts are similar between the two groups then the variability behavior of the ensembles in the observer's frame will be directly comparable. The difference in magnitude range between the samples affects $V$ because the measurement error $\sigma$ is dependent on the object magnitude. We therefore do not compare all of the CRATES candidates covered by Palomar-QUEST (1,917 objects) with all of the 278 FSRQs, but subsets of each with statistically equivalent magnitude distributions: 866 CRATES candidates and 260 FSRQs. The $V$ distributions of these subsets are shown in figure 11, with the CRATES objects shown as solid lines and the FSRQs as dashed lines. The time lag bin size increases with time lag so that the longer time lag histograms are minimally sensitive to small differences in the objects' redshift distributions. The plots are each normalized to a total of 100 objects to facilitate comparisons. Clearly, the variability timescales and amplitudes of the CRATES objects and the FSRQ sample are qualitatively similar. To quantify whether the $V$ s of the two samples come from the same underlying distribution, we compare the data using a K-S test for each time lag interval. Because the K-S test is sensitive to the shape of the distribution, we ignore datapoints with $[m(t)-m(t-\tau)]^{2}<\sigma^{2}$ since our arbitrary assignment of $V=0$ for these measurements artificially modifies the distribution. The probabilities that the $V$ data come from the same distributions range from $4 \%$ to $26 \%$, with a median of $12 \%$. The optical variability behavior of the CRATES objects and the FSRQs is therefore similar on all timescales measured. Any differences in the redshift distributions between the CRATES and the FSRQ samples will cause the distributions to differ, so the measured probabilities can be taken as lower limits. The similarity we see in optical variability properties between the CRATES sample and known FSRQs provides evidence that the CRATES objects are dominated by FSRQs similar to those found using a variety of radio and X-ray methods.

The CRATES sample has a small but significant overlap with objects spectroscopically identified as quasars by SDSS. The quasar sample used in figures 3, 4, and 7, and described in detail

\begin{tabular}{|l|l|}
\hline$\beta_{\text {app }}$ & Median $V$ \\
\hline 0 to 7 & $0.09 \pm 0.06$ \\
7 to 18 & $0.17 \pm 0.12$ \\
18 to 27 & $0.30 \pm 0.25$ \\
\hline
\end{tabular}

Table 2: Median variability $V$ for FSRQs with $0<\beta_{a p p} \leq 7,7<\beta_{a p p} \leq 18$, and $18<\beta_{a p p} \leq 27$. 
in Paper I, is comprised of $\sim 23,000$ predominantly UV-selected objects from SDSS Data Release 5 (Adelman-McCarthy et al. (2007)). The objects are optically spectroscopically confirmed to be quasars; however the presence of broad emission lines in both type I quasars and FSRQs can lead to overlapping identifications. There are 83 objects common to the CRATES and type I quasar samples. Thirty-eight additional CRATES objects are in the SDSS DR5 spectroscopic quasar list, but were removed from the Paper I data set due to either their presence in other blazar samples (36 objects) or their low redshift (2 objects). Therefore, $6 \%$ of the CRATES sample observed by QUEST has been seen by SDSS to have quasar-like optical colors and spectra. This does not disqualify the objects from being FSRQs, but illustrates the difficulty of distinguishing between AGN classes and highlights the influence of selection effects on samples of AGN. The CRATES objects make up $0.4 \%$ of the quasar sample used in this work and Paper 1 . This order of contamination will not affect the average variability properties of the type I quasar ensemble.

\subsection{Variability Duty Cycle}

About $15 \%$ of the blazars' $V$ measurements are greater than 0.4 . Are these measurements due to each object varying dramatically during $15 \%$ of its measurements, or a small fraction of the blazars varying with high amplitude all the time, or something between? Figure 12 shows, for FSRQ, BL Lac, CRATES, and quasar samples including only objects with at least $10 \mathrm{~V}$ measurements, the fraction of an object's $V$ measurements that are greater than 0.4. Each histogram is normalized to a total of 100 objects. $60 \%, 65 \%$, and $69 \%$ of the BL Lacs, FSRQs, and CRATES objects, respectively, have no $V$ values greater than 0.4 . Those for which we do measure such high variability are evenly distributed up to a measurement fraction of roughly 0.8: e.g. equal numbers of blazars are highly variable across $10 \%$ of our measurements as are highly variable across $70 \%$ of them. On the other hand, we see $94 \%$ of quasars always with $V \leq 0.4$; those which do appear highly variable tend to show such variability across fewer than $\sim 40 \%$ of their measurements. These results reinforce the conclusion that blazars have a large range of behavior; they show active and quiescent phases on all timsecales spanned by our survey. The majority of blazars, however do not contribute to the highest levels of variability seen by the survey.

We caution that without close, even spacing of observations it is not possible to determine objects' intrinsic duty cycles. By selecting objects with at least 10 measurements we ensure that the blazars studied here are observed on a range of timescales over at least two years. However, by the nature of a ground based survey, there are months at a time during which we have no observations of a particular blazar. We therefore cannot say that a blazar varies $90 \%$ of the time, only over $90 \%$ of our measurements. This analysis describes the variety of variability behavior observed in blazars over timescales from hours to several years, and is the best approximation to an intrinsic duty cycle measurement as can be made with such a survey. 


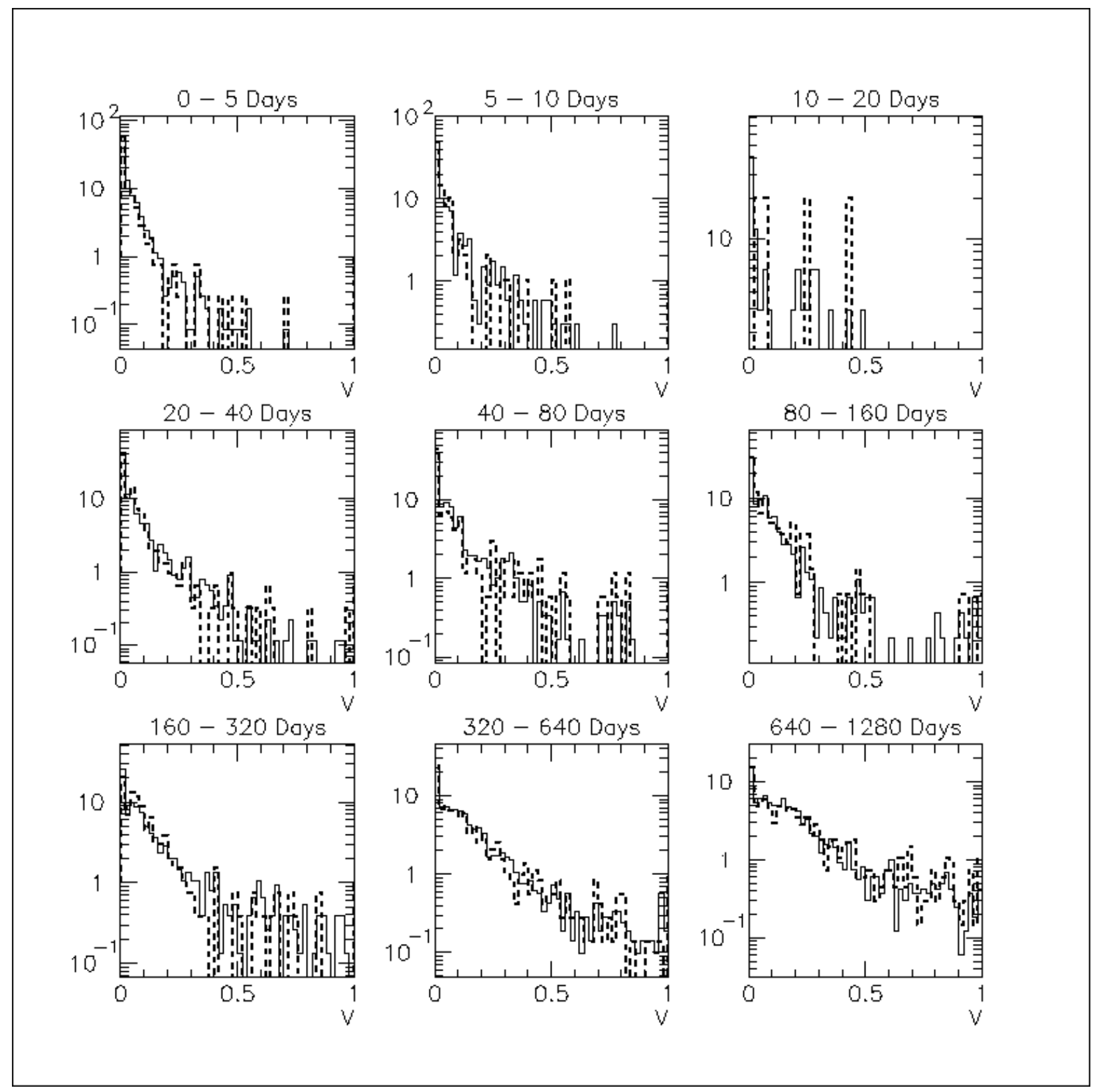

Fig. 11. - Variability $V$ histograms of CRATES objects (solid line) and FSRQs evaluated at $\mathrm{z}=0$ (dashed line) for 9 different time lag bins. 


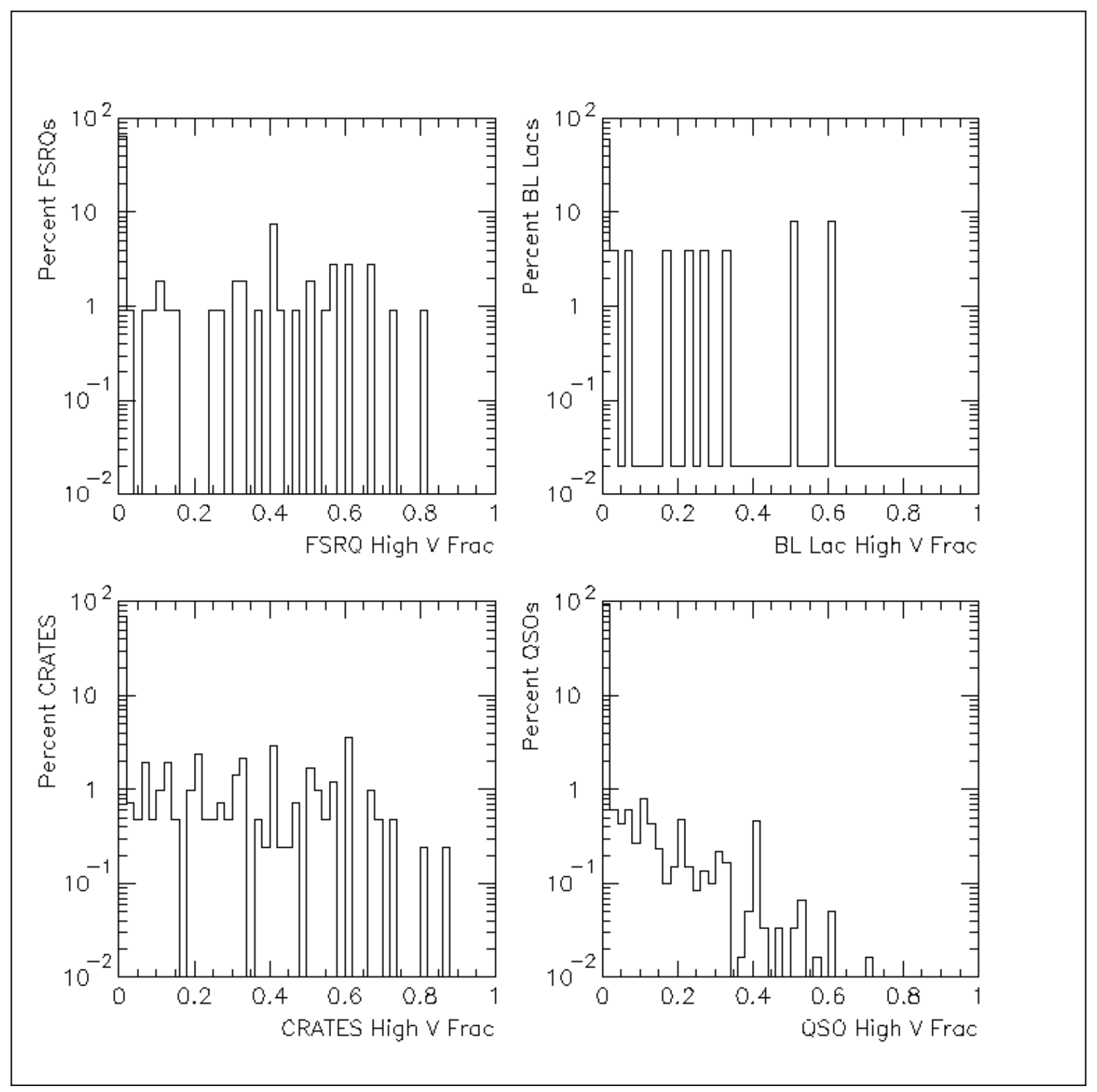

Fig. 12.- Fraction of $V$ measurements greater than 0.4, for objects with at least 10 measurements. Number of objects on the $\mathrm{Y}$ axis is normalized to a total of 100 in each plot. 


\section{Discussion}

Previous variability studies of blazars have used many, frequent measurements of one or a few objects to examine the details of individual fluctuations in the sources. In this work we consider a few measurements of many blazars in order to study the common variability properties of the class as a whole. Our results reflect the ensemble behavior of a diverse population. The choppy nature of the FSRQ and BL Lac structure functions suggests that blazar variability differs widely from object to object. This is also shown by the wide range of high variability duty cycles seen in the objects. About $35 \%$ of the blazars are seen to vary by $V>0.4$ during the 3.5 years of the Palomar-QUEST survey. These highly variable objects have a roughly continuous range of high variability duty cycles up to about $80 \%$ of the measurements, showing that while some blazars vary drastically over nearly all observations, others show active periods with no single characteristic length within the time span of our survey. Because our blazar samples show such diversity, the ensemble variability properties cannot be interpreted as describing the behavior of individual objects. Instead, they highlight variability amplitudes and timescales that are common in BL Lacs and FSRQs, and illustrate differences in typical behaviors between classes.

We see that blazars show some variability properties similar to those of type I quasars. Specifically, the majority of the variability takes place at amplitudes below a few tenths of a magnitude, and the low-amplitude peak of variability broadens with increasing time lag. Both FSRQs and BL Lacs show a higher amplitude tail superimposed on this low amplitude peak; in FSRQs the quasar-like peak is more prominent. This similarity between FSRQs and quasars may be related to their spectral similarity: the presence of thermal emission, including broad emission lines. Accretion disk flux, partly reprocessed in the broad line region, may be responsible for much of the low amplitude variability seen in FSRQs. The flatter shape of the BL Lac variability distribution compared to the FSRQs suggests that while accretion disk emission may also play an important role in BL Lac variability, its contribution is less significant than in FSRQs.

The highest amplitude variability, with $V \gtrsim 1$ magnitude, is more common in FSRQs than BL Lacs at all timescales. High amplitude blazar variability is commonly attributed to shock dissipation in the jet; the fact that the strongest variability differs between FSRQs and BL Lacs implies different jet characteristics for the two groups. Indeed, there is strong evidence, independent of variability, that FSRQs and BL Lacs have different jet energies and morphologies: BL Lacs are thought to be intrinsically FR I radio galaxies, and FSRQs to be FR IIs. The higher energy jets characteristic of FR IIs may be important in generating the excess high amplitude variability observed in FSRQs.

Blazars exhibit a wide variety of jet energies, with differences due to intrinsic properties as well as beaming direction. For example, the apparent velocity of blazar jets in the radio band has

been seen to range from stationary to over $\sim 30$ times the speed of light! (Cohen et al. (2007)) 31 of the FSRQs in our sample have been monitored by MOJAVE and have maximum jet speed measurements. Those FSRQs with faster jets show more high-amplitude variability than those 
with slower jets. This could be because the faster radio regions generate shocks which create more optical flux; in this case the radio and optical properties would be directly related, generated in the same region of the jet. Or, the radio and optical flux could be indirectly related, with the powerful injections which fuel the radio speed also enhancing optical variability elsewhere in the jet.

The structure function is essentially the clipped mean of the variability amplitude histogram, plotted versus rest frame time lag, and has often been used to study AGN variability. The FSRQ and BL Lac structure functions have shapes unlike that of type I quasars, showing excess variability on both short and long timescales. The blazars' structure functions reinforce the conclusion that FSRQ variability is more similar to quasars' than BL Lac variability is to quasars', particularly at timescales of roughly 70 to 250 days. The FSRQ structure function shows a clear excess of power compared to type I quasars around timescales of 20 to 50 days. This detection is consistent with reports of flares with similar timescale in objects like GC $0109+224$ (Ciprini et al. (2003)). However, this power excess can not be a result of flares at that timescale in all blazars, as that kind of recurrent, well-behaved fluctuation would consistently lead to a smooth, monotonic structure function shape that is qualitatively different from the data. Equivalently, we can see in figure 3 that on these timescales there is increased high amplitude variability. However, such variability is not absent at other timescales, even at those where the FSRQ and quasar structure functions are similar.

The CRATES Survey (Healey et al (2007)) has identified a very large number of FSRQ candidates by their radio emission. The variability amplitude distributions of these candidates as seen in the Palomar-QUEST Survey are similar to those of confirmed FSRQs at all measured time lags. The CRATES selection criteria are independent of variability; the similarity of their optical variability to that of FSRQs therefore implies that the CRATES objects are likely to be predominantly FSRQs. These candidates promise to be an extremely valuable set from which to study the ensemble properties of blazars, particularly if redshifts of the objects can be obtained so that more accurate timescales of variability may be measured.

For this work we have divided the blazar sample into BL Lacs and FSRQs because there are significant differences in the optical spectral shape between the two classes, likely related to jet morphology and differences in the host radio galaxy. It may be helpful to divide the blazar sample in different ways that could be more in line with their different variability properties. For example, BL Lacs can be divided into HBL and LBL groups (high peaked and low peaked BL Lacs) according to the central frequencies and relative heights of the synchrotron and Compton peaks in the SED. FSRQs typically have SED peak frequencies similar to LBLs, although some have been seen with SEDs more like HBLs' (Padovani et al. (2007)). It is not clear how the HBL/LBL status of the blazar relates to the object's fundamental properties. It has been proposed that HBL jets may be dominated by synchrotron self-Compton (SSC) shock dissipation while the LBLs may follow external radiation Compton (ERC) mechanisms. This is supported by the fact that FSRQs, which tend to have SEDs similar to LBLs, exhibit broad optical emission lines; the presence of this thermal emission is consistent with the ERC model's invocation of disk and broad emission line 
radiation acting as Compton seed photons (Fossati et al. (1998)). The HBL/LBL dichotomy is one example of a different blazar categorization that may yield optimally contrasting, and therefore illuminating, variability results. Such rebinning of the data awaits further multiwavelength and variability information to provide sufficient statistics.

\section{Conclusions}

We have used the Palomar-QUEST Survey to examine the ensemble optical variability of 276 FSRQs and $86 \mathrm{BL}$ Lacs. This is the first study of the common variability characteristics of so many of these rare AGN. We have therefore described the variability in several ways, showing distributions of variability amplitudes at numerous time lags as well as a more traditional structure function analysis.

We find that blazars have a wide range of behavior from object to object. The diversity of behavior within the blazar class is qualitatively apparent in the shape of the ensemble structure functions. We also see that the duty cycle of blazars differs widely from object to object. About the same fraction of objects show high amplitude variability across $\sim 5 \%$ to $\sim 80 \%$ of their measurements. Roughly $65 \%$ of blazars do not show this high amplitude variability ( $\Delta m \gtrsim 0.4$ magnitudes) at all during the survey. Therefore, while optical variability may be a distinctive feature of blazars, details of the variability appear very different between objects on timescales up to a few years. The ensemble variability of the class, studied using few measurements of many objects, therefore reflects behavior characteristic of the group as a whole rather than descriptive of individual members.

Both BL Lac and FSRQ classes have similarities in variability amplitudes and timescales compared to type I quasars, however with more fluctuations superimposed at all timescales measured by Palomar-QUEST. FSRQs show more similarities to type I quasars than BL Lacs do, implying a stronger role for accretion disk variability in FSRQs. The heightened presence of disk variability is in accordance with the presence of broad emission lines in FSRQ spectra, which are absent in BL Lacs. Periodicity is not observed to be a common variability property in blazars.

FSRQs exhibit more variability at the highest amplitudes than BL Lacs. This is likely related to higher jet energies in FSRQs compared to BL Lacs. For a subset of 31 FSRQs with measured maximum apparent jet speeds in the radio band, the radio jet speed correlates positively with the optical variability amplitude.

CRATES candidate FSRQs show similar variability amplitudes and timescales to the confirmed FSRQs. This behavior is independent of the CRATES selection criteria, and is therefore a good test to show that this sample is likely dominated by FSRQs similar to those known.

The discovery of new blazars, for example from the CRATES survey, is very important for the thorough study of this rare class. Characterizing the appearance of blazars in sparsely sampled, wide field surveys such as Palomar-QUEST facilitates the optical variability-based selection of new 
blazars. With more statistics we may be able to divide the class into subsets that better clarify the processes behind the dramatic variability.

We thank S. G. Djorgovski and his group for helpful discussions. This work is supported by the Office of Science of the Department of Energy and the National Science Foundation.

This research has made use of data from the MOJAVE (Lister and Homan, 2005, AJ, 130, 1389), 2cm Survey (Kellermann et al., 2004, ApJ, 609, 539), and CRATES (Healey et al., 2007, ApJS, 171, 61) programs.

Facilities: PO:1.2m 


\section{REFERENCES}

Adelman-McCarthy, J. et al. 2007, ApJS, 172, 634

Andrews, P. et al. 2008, PASP, 120, 703

Baltay, C. et al. 2007, PASP, 120, 703

Bauer, A. et al. 2009, ApJ, 696, 1241

Bonning, E.W. et al. 2009, arXiv:0812.4582v1, Submitted to ApJ

Chatterjee, R. et al. 2008, ApJ, 689, 79

Ciaramella, A. et al. 2004, A\&A, 419, 485

Ciprini, S. et al. 2003, A\&A, 400, 487

Cohen, M. et al. 2007, ApJ, 658, 232

Collier, S. and Peterson, B. 2001, ApJ, 555, 775

Collinge, M. et al. 2005, AJ, 129, 2542

D'Arcangelo, F. et al. 2007, ApJ, 659, L107

Donato, D. et al. 2005, A\&A, 433, 1162

Fanaroff, B. and Riley, J. 1974, MNRAS, 167, 31

Fossati, G. et al. 1998, MNRAS, 299, 433

Healey, S. et al. 2008, ApJS, 175, 97

Healey, S. et al. 2007, ApJS, 171, 61

Heidt, J. and Wagner, S. 1996, A\&A, 305, 42

Hewitt, A. and Burbidge, G. 1993, ApJS, 87, 451

Kartaltepe, J. and Balonek, T. 2007, AJ, 133, 2866

Li, H. and Kusunose, M. 2000, ApJ, 536, 729

Lindfors, E.J. et al. 2006, A\&A, 456895

Lister, M. and Homan, D. 2005, ApJ, 130, 1389

Marscher, A. et al. 2008, Nature, 452, 966

Massaro, E. et al. 2007, AIP Conference Proceedings, 921, 349 
Metcalf, R. and Maglicchetti, M. 2006, MNRAS, 365, 101

Monet, D. et al. 1998, Bulletin of the American Astronomical Society, 38, 1427

Mücke, A. and Protheroe, R.J. 2001, Astroparticle Physics, 15, 121

Padovani, P. et al. 2007, ApJ, 662, 182

Peterson, B.M. 1997, An Introduction to Active Galactic Nuclei (Cambridge University Press)

Raiteri, C.M. et al. 2008, A\&A, 491, 755

Sowards-Emmerd, D. et al. 2005, ApJ, 626, 958

Stickel, M. et al. 1991, ApJ, 374, 431S

Turriziani, S. et al. 2007, A\&A, 472, 699

Urry, C. and Padovani, P. 1995, PASP, 107, 803

Vanden Berk, D. et al. 2004, ApJ, 601, 692

Veron-Cetty, M. and Veron, P. 2006, A\&A, 455, 773 